\title{
Ligand-Based Virtual Screening for the Discovery of Inhibitors for Protein Arginine Deiminase Type 4 (PAD4)
}

Chian Ying Teo', Mohd Basyaruddin Abdul Rahman¹, Adam Leow Thean Chor², Abu Bakar Salleh ${ }^{3}$, Pedro J Ballester ${ }^{4}$ and Bimo A Tejo ${ }^{*}$

${ }^{1}$ Department of Chemistry, Faculty of Science, Universiti Putra Malaysia, 43400 UPM Serdang, Malaysia

${ }^{2}$ Department of Cell and Molecular Biology, Faculty of Biotechnology and Biomolecular Sciences, Universiti Putra Malaysia, 43400 UPM Serdang, Malaysia

${ }^{3}$ Department of Biochemistry, Faculty of Biotechnology and Biomolecular Sciences, Universiti Putra Malaysia, 43400 UPM Serdang, Malaysia

${ }^{4}$ European Bioinformatics Institute, Wellcome Trust Genome Campus, Hinxton, Cambridge, CB10 1SD, United Kingdom

\begin{abstract}
Protein Arginine Deiminase type 4 (PAD4) is a new therapeutic target for the treatment of rheumatoid arthritis. In this study, ligand-based virtual screening with the integration with drug repurposing strategy was applied to the discovery of PAD4 inhibitors. Ultrafast Shape Recognition (USR) was used to search for compounds with similar shape to a previously reported inhibitor with harmful side-effects, i.e., streptonigrin. Thirty five lead-like compounds and two existing drugs were obtained from virtual screening and their inhibitory activity was tested at fixed concentration of $100 \mu \mathrm{M}$. Five lead-like compounds showed significant inhibition on the enzymatic activity of PAD4. The potency of the best compound was investigated by carrying out IC50 study. Importantly, the structure of the best of these new active molecules was strikingly different from that of streptonigrin. Furthermore, this new PAD4 inhibitor is the most potent to date found by a computational approach and its structure can be optimized in the future for the design of an even better inhibitor of PAD4.
\end{abstract}

Keywords: Protein Arginine Deiminase type 4; citrullination; Histagged protein; High-Throughput Screening

\section{Introduction}

Protein arginine deiminase, also known as Peptidyl Arginine Deiminase (PAD) is an enzyme catalyzing post translation conversion of arginine to citrulline. It is a $\mathrm{Ca}^{2+}$ dependent enzyme; without $\mathrm{Ca}^{2+}$, it cannot perform the conversion properly due to improper structural rearrangements for substrate binding [1]. There are five isoforms of human PADs, which are PAD1 to PAD4 and PAD6. Each isoform has their own physiological roles in different locations in human body. For example, PAD4, which is localized in both cytoplasmic granules and nucleus, is expressed mainly in immune system cells. One of its physiological roles in human and also other mammal's bodies is cellular differentiation. While PAD1 and PAD3 are playing role in skin differentiation, PAD2 has putative role in brain development and PAD6 is important in embryonic development [2].

Dysregulated activity of PAD, especially PAD4 has been linked to the onset and progression of diseases such as rheumatoid arthritis, multiple sclerosis and cancer. Several researchers have argued that the dysregulation of PAD4 is the main cause for the onset of rheumatoid arthritis [3-5]. The citrullination process catalysed by PAD4 produces citrullinated protein, which is the main target of the immune system. Consequently, PAD4 constitutes a promising drug target for the treatment of rheumatoid arthritis. Several studies have been carried out to discover new PAD4 inhibitors by taking different approaches [6-11]. Drug discovery is always a time and cost consuming process. An effective and efficient approach for finding good inhibitors with minimum time and cost is required. With the aid of advanced computational method, molecular bioactivity of compounds can be predicted and potential drugs can be found in a large database containing millions or even billions of screening compounds with reduced time scales and financial costs [12]. The integration method of conventional High-Throughput Screening (HTS) with the computational methodology namely virtual High-Throughput Screening (vHTS) accelerates the drug discovery process. Several strategies can be applied in finding suitable drugs from more than millions of compounds such as structure-based and ligand- based drug design. Following from the development of numerous algorithms, including pharmacophore searching and high- throughput docking methods, drug discovery with vHTS as a starting point has gained momentum [13].

The availability of the large number of crystal structures of the target protein in Protein Data Bank encourages the application of structurebased drug design. However, previous applications of structure-based virtual screening techniques have only provided $\mathrm{mM}$ inhibitors of this target [14]. Alternatively, ligand-based techniques represent an attractive strategy in searching of drug candidates [15]. Here we investigate whether a ligand-based approach is a better strategy for this target. The technique searches suitable candidates for the drug target by comparing the molecular shape of the compounds in the database with a given query molecule. The template can be a known inhibitor of a target protein, a natural product or even a patented chemical [16]. We integrated the technique with drug repurposing strategy in order to search PAD4 inhibitors from drugs that have already proven to be safe for human.

A necessary condition for the biological activity of a molecule is its complementarity in shape to shape the binding site of the receptor [1719]. Such complementarity is important for the ligand and receptor atoms involved to be sufficiently close to enable interactions such as hydrogen bonding and hydrophobic interaction [15]. Another

*Corresponding author: Bimo A Tejo, Department of Chemistry, Faculty of Science, Universiti Putra Malaysia, 43400 UPM Serdang, Malaysia, Tel: +62-21-5465496; Fax: +62-21-70200272; E-mail: bimo@upm.edu.my, bimo.tejo@surya.ac.id

Received May 02, 2013; Accepted June 26, 2013; Published July 03, 2013

Citation: Teo CY, Abdul Rahman MB, Thean Chor AL, Salleh AB, Ballester PJ, et al. (2013) Ligand-Based Virtual Screening for the Discovery of Inhibitors for Protein Arginine Deiminase Type 4 (PAD4). Metabolomics 3: 118. doi:10.4172/21530769.1000118

Copyright: (c) 2013 Teo CY, et al. This is an open-access article distributed under the terms of the Creative Commons Attribution License, which permits unrestricted use, distribution, and reproduction in any medium, provided the original author and source are credited. 
Citation: Teo CY, Abdul Rahman MB, Thean Chor AL, Salleh AB, Ballester PJ, et al. (2013) Ligand-Based Virtual Screening for the Discovery of Inhibitors for Protein Arginine Deiminase Type 4 (PAD4). Metabolomics 3: 118. doi:10.4172/2153-0769.1000118

advantage of finding molecules with similar shape in a database is that no specification of chemical structure such as the types of atom or their bond arrangements is made, so there is no limitation about only the compounds with same atoms will be selected, in contrast the compounds with different chemical scaffold but with similar molecular shape, can be found [16]. The ability is known as scaffold hopping [20], which is very valuable in discovery of non-intuitive novel drug leads.

In this study, potential inhibitors for PAD4 were searched from databases by comparing the molecular shape similarity with a reported inhibitor by using Ultrafast Shape Recognition (USR) [16]. In addition to drug-like compounds, marketed drugs were also present in the screened database. Finding a new target for existing drugs is not a new approach in pharmaceutical industry. Repurposing of an existing drug to other targets, diseases, pathways or functions is a good strategy for speeding up the process of drug discovery. By considering approved drugs that have already been shown to be safe in humans, off-target associations of such drugs may result in shortcuts to Phase II trials for the new indications [21].

USR is based on the observation that the shape of a molecule is uniquely determined by the relative position of its atoms. It can compare the molecular shape effectively in a large database with thousand times faster than pre-existing methods [22]. In the past, USR alone has been shown to be particularly useful for prospective virtual screening [12,23]. The given molecule used for similarity search is streptonigrin (Figure 1) which is the reported inhibitor discovered by Knuckley et al. [9]. It was found to be a potent, selective and irreversible PAD4 inactivator but it is not suitable to be utilized as a useful PAD4 probe or a therapeutic. This is due to exhibiting pleiotropic effects on several cellular processes and also possessing a number of side effects such as hair loss, nausea, bone marrow depression and high toxicity which have limited its use in cancer treatment [24]. Therefore, the main objective of this study is to find alternative PAD4 inhibitors that could lead to drug leads with better pharmacological properties.

\section{Materials and Methods}

\section{Expression and purification of PAD4}

PAD4 was expressed as described in previous study [14]. PAD4 was overexpressed as His- tagged protein in BL21 (DE3) cells carrying the expression plasmid. The purified PAD4 was obtained by applying the fusion protein to Ni-Sepharose ${ }^{\text {Tax }} 6$ Fast Flow affinity column (GE Healthcare, Uppsala, Sweden). Detailed purification protocol was also described in previous publication [14]. The presence of the protein

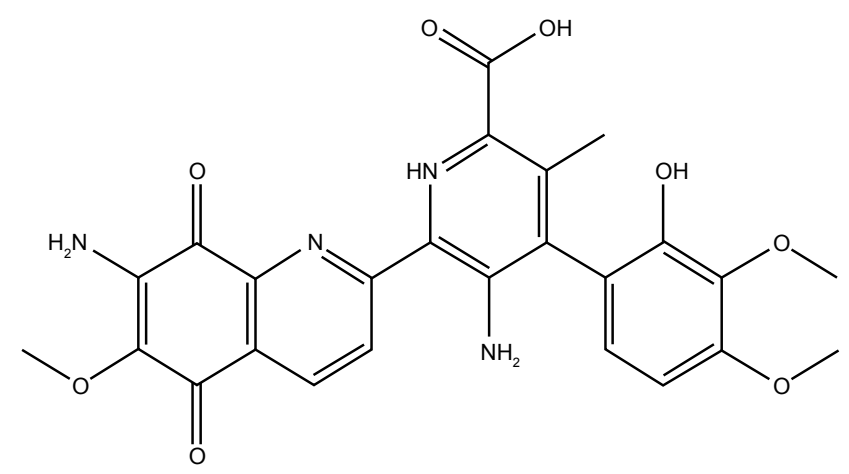

Figure 1: Chemical structure of streptonigrin. in specific fractions was detected by SDS-PAGE analysis. Fractions containing the protein were pooled and stored at $4^{\circ} \mathrm{C}$.

PAD4 inhibition assay-quick screening: Quick screening was carried out in the manner identical to the method used in previous study [14]. $0.06 \mu \mathrm{M}$ of PAD4 was pre-incubated with $100 \mu \mathrm{M}$ of inhibitors in the reaction buffer solution containing $100 \mathrm{mMTris}(\mathrm{pH}$ 7.2), $10 \mathrm{mM}$ calcium chloride and $10 \mathrm{mM}$ DL-dithiothreitol at $37^{\circ} \mathrm{C}$ for 15 minutes. The reaction was started by adding the substrate, 10 $\mathrm{mM} \mathrm{N}-\alpha$ - benzoylarginine ethyl ester (BAEE) and the reaction mixture was then incubated at $37^{\circ} \mathrm{C}$ for 15 minutes. The enzymatic reaction was terminated by adding $60 \%(\mathrm{w} / \mathrm{v})$ perchloric acid. For color development, redox reagent prepared by ferrous ammonium sulfate hexahydrate and ammonium iron (III) sulfate dodecahydrate in $1 \mathrm{~N} \mathrm{H}_{2} \mathrm{SO}_{4}$ was added to the reaction mixture, then boiled for 10 minutes. After cooling down to room temperature, the acid mixture (phosphoric acid, sulfuric acid and deionized water) and $12.5 \mathrm{mM}$ of 2,3-butanedione monoxime solution was added. It was boiled for 20 minutes then cooled. The absorbance at $490 \mathrm{~nm}$ was measured and compared to a citrulline standard curve to determine the concentration of citrulline produced during the course of the reaction.

$\mathrm{IC}_{50}$ study: PAD4 was pre-incubated with various concentration of the inhibitor in the reaction conditions as described above. The method of performing $\mathrm{IC}_{50}$ study is as described previously [14]. The relative $\mathrm{IC}_{50}$ value was calculated by fitting the data to standard $\mathrm{IC}_{50}$ equation in the Grafit [25] program.

Virtual screen: The screening database was generated from ZINC8 subset 1 of all usual ligands ( $\mathrm{pH} 5.75$ to 8.25) downloaded from http:// zinc8.docking.org/, which contains 3,472,461 lead-like commerciallyavailable molecules. Each of these molecules is already protonated and provided as a single three-dimensional conformer. Multiple threedimensional conformers were calculated for each molecule using Omega 2.4.3 working with default settings. The latter led to $641,485,760$ conformers that is an average of 185 conformers per molecule. Once the USR descriptors were calculated and loaded in memory, USR took 57 seconds to search the $641,485,760$ conformers for those similar to streptonigrin (USR-score $<0.87$; using single core in a workstation with Intel Core2 Duo CPU@2.66GHz, 2MB RAM).

\section{Molecular docking}

Molecular docking was performed using AutoDock Vina. AutoDock Tools (ADT) was utilized to set the size and the center of the grid box, and to prepare the input .pdbqt file for the PAD4 structure (PDB 2DWE) and the ligands (streptonigrin and the most potent compound in this study). The grid box cavity was set at 21.295, 41.956, 22.264 (in the dimension of $x, y, z)$ with the grid size of $20 \times 30 \times 20$ using $1.000 \mathrm{~A}^{0}$ spacing. The predicted binding affinity $(\mathrm{kcal} / \mathrm{mol})$ was calculated based on the scoring function used in AutoDock Vina. LigPlot program was used to analyze the molecular interaction between ligands and PAD4.

\section{Results and Discussion}

After USR highlighted a number of compounds as hits, a quick experimental screening was carried out to identify the inhibitory activity of the compounds. Due to the availability of the compounds, from the 45 USR hits, only 37 of them were purchased for further investigation. Thirty five of them (compounds 1-35) are drug-like compounds while the remaining are marketed drugs, dofetilide (compound 36) and lumefantrine (compound 37). The quick screening was performed by carrying out the inhibition assay. The activity of PAD4 after inhibition was compared with the activity of PAD4 without 
Citation: Teo CY, Abdul Rahman MB, Thean Chor AL, Salleh AB, Ballester PJ, et al. (2013) Ligand-Based Virtual Screening for the Discovery of Inhibitors for Protein Arginine Deiminase Type 4 (PAD4). Metabolomics 3: 118. doi:10.4172/2153-0769.1000118

any inhibitor present. The concentration of each inhibitor was fixed at $100 \mu \mathrm{M}$ and the percentage of PAD4 remaining activity after adding inhibitor was calculated [14]. Streptomycin [8] and streptonigrin [9] were used as positive controls in the screening and their $\mathrm{IC}_{50}$ values were determined. $\mathrm{IC}_{50}$ for streptomycin was identical as the reported value, i.e. $1.8 \mathrm{mM}[8]$ while for streptonigrin was approximately $40 \mu \mathrm{M}$ which is higher than the reported value, i.e. $1.87 \mu \mathrm{M}$ [9]. The variations are not uncommon for absolute inhibition in different assay systems.

Figure 2 shows the percentage of PAD4 remaining activity with the presence of the compounds. With the concentration of $100 \mu \mathrm{M}$, out of the 37 compounds, five compounds which are compound $2,4,5,11$ and 30 exhibited significant inhibition to PAD4. The compounds inhibited PAD4 by more than $10 \%$ at $100 \mu \mathrm{M}$ (significant difference; $p<0.05$ ). The chemical structures of the most potent compounds are showed in Figure 3 . It is worth noting that these are chemically diverse structures, which bear little resemblance with the search template (streptonigrin).

All of the five potential compounds were drug-like compounds but regrettably, dofetilide and lumefantrine did not exhibited significant inhibition to PAD4. Dofetilide is Food and Drug Administration

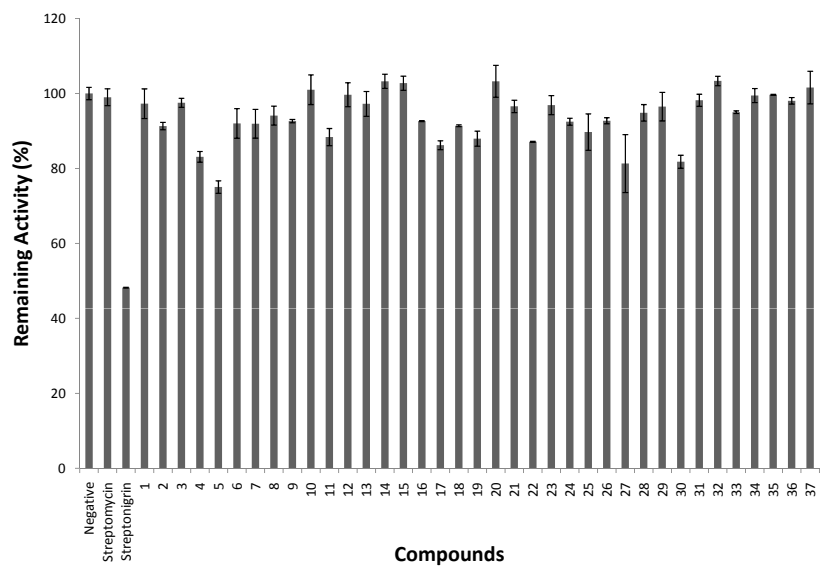

Figure 2: Percentage of remaining activity of PAD4 in the presence of $100: M$ inhibitors.

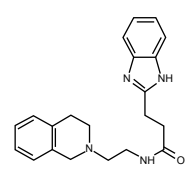

(a)

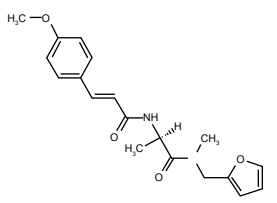

(e)

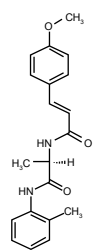

(b)

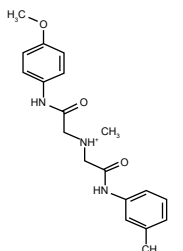

(d)

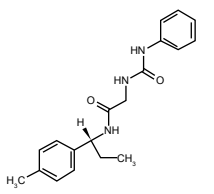

(e)
Figure 3: Structure of compounds that exhibited significant inhibition to PAD4 Compound 2 (a), compound 4 (b), compound 5 (c), compound 11 (d), and compound $\mathbf{3 0}(\mathrm{e})$.
(FDA) approved drug for the maintenance of normal sinus rhythm [26] while lumefantrine is an antimalarial agent. Utilizing existing drugs for new indications is a beneficial method because it will increase the success rate and reduce the costs and time for drug development [20] The combination of computational approach with drug repurposing seems to be an attractive technique in drug discovery as it accelerates the screening process. It enables the screening of large database of marketed drugs to a new target. Although USR identified dofetilide and lumefantrine as compounds similar with streptonigrin, unfortunately, they did not inhibit PAD4 significantly. Although these new PAD4 inhibitors arised from a similarity search using streptonigrin as template, their chemical structures are remarkably different from that of streptonigrin. The ability to scaffold hop is a very desirable trait of virtual screening tools. Scaffold hopping was a concept coined by Schneider et al. [27] in 1999 as a technique to discover isofunctional molecular structures with significant different molecular core structures. With the application of the strategy in drug design process, it will definitely contribute to the discovery of new class of drug for a particular disease. Scaffold hopping is also widely used in lead optimization approaches $[28,29]$. When the scaffold of the compound is modified, some of the undesired properties will be eliminated. Historically, quite a number of marketed drugs are derivative of natural products, natural hormones or other drugs through scaffold modification [30]. The earliest example of scaffold hopping is tramadol with reduced side effects compared to its parent compound, morphine [30]. The parent compound in this study, streptonigrin possesses numerous of side effects that forbid it from becoming drug for rheumatoid arthritis treatment although it inhibits PAD4 excellently, with IC50 value of $1.87 \pm 0.24 \mu \mathrm{M}$ [9]. In our study, we aimed at investigating whether USR could identify PAD4 inhibitors with a different chemical scaffold than streptonigrin, which may therefore lead to fewer side-effects to the patients.

After the quick experimental screening, we intended to further study the potency of the potent compounds in inhibiting PAD4 by performing $\mathrm{IC}_{50}$ study. Unfortunately, compound 2, 4, 11 and 30 are weak inhibitors with $\mathrm{IC}_{50}$ values exceeding $500 \mu \mathrm{M}$. The $\mathrm{IC}_{50}$ values for the compounds cannot be determined accurately due to the low solubility of the compounds in the reaction buffer solution. They reprecipitated when added at high concentration in the reaction buffer solution. The solubility problem limits the potency of the compounds to be further investigated. Furthermore, they do not possess the property of a good drug as a good drug always has good solubility in aqueous solution since drug with poor aqueous solubility tends to have absorption problems since the flux of drugs across the intestinal membrane is proportional to concentration gradients between the intestine lumen and blood [31].

Out of the five compounds, only compound $\mathbf{5}$ exhibited good solubility in the reaction buffer solution. This compound showed the highest inhibition to PAD4 in quick screening, reducing PAD4 activity by $25 \%$ in $100 \mu \mathrm{M}$ concentration. The IC $_{50}$ value of compound $\mathbf{5}$ was determined by inhibition assay using various concentration of the compound. Figure 4 shows the dose- response curve of the compound. From concentration 512 to $700 \mu \mathrm{M}$, the activity of PAD4 was only slightly decreased from 45 to $41 \%$, most likely this is the maximum effect of Compound 5. The inhibitory effect of the compound could not be examined further as the compound started to reprecipitate at concentration higher than $700 \mu \mathrm{M}$. The absolute $\mathrm{IC}_{50}$, which is the concentration of inhibitor that brings the remaining activity down to $50 \%$, was estimated from the graph. The estimated absolute $\mathrm{IC}_{50}$ of compound 5 was $300 \mu \mathrm{M}$, while the relative $\mathrm{IC}_{50}$ value calculated by Grafit [25] using its $\mathrm{IC}_{50}$ standard equation is $200 \mu \mathrm{M}$. Although the 
Citation: Teo CY, Abdul Rahman MB, Thean Chor AL, Salleh AB, Ballester PJ, et al. (2013) Ligand-Based Virtual Screening for the Discovery of Inhibitors for Protein Arginine Deiminase Type 4 (PAD4). Metabolomics 3: 118. doi:10.4172/2153-0769.1000118

value is higher than the $\mathrm{IC}_{50}$ value of streptonigrin, it can be considered as a moderate inhibitor for PAD4 if compared with streptomycin, tetracycline and methotrexate, whose $\mathrm{IC}_{50}$ values are $1.8 \pm 0.3 \mathrm{mM}$, $0.78 \pm 0.14 \mathrm{mM}$ and $>10 \mathrm{mM}$, respectively [8]. Compound $\mathbf{5}$ is more potent than compounds discovered by structure-based strategy in previous study [24]. The $\mathrm{IC}_{50}$ value of the best compound discovered in the structure-based study is $1.5 \mathrm{mM}$, which is five-folded compared with compound 5. Molecular docking result suggests that compound 5 has favorable interaction with PAD4 as indicated by its binding affinity, i.e., $-5.8 \mathrm{kcal} / \mathrm{mol}$. This compound forms one hydrogen bond between oxygen $\mathrm{O}_{3}$ of the carbonyl group and the hydroxyl group of Ser406 side chain. The main interaction that governs the binding of compound 5 to PAD4 is hydrophobic contacts, which mostly occur between the furan ring of compound 5 and Trp347, Ile401, Gly403, His471, His640, Gly641, and Cys645. There is one hydrophobic contact between the aromatic ring of compound 5 with Gly399 on the surface of PAD4 binding pocket. It is interesting to note that the furan ring of Compound 5 makes a contact with Cys645, a catalytic residue that has been suggested as a target in designing the covalent inhibitors for PAD4 [6]; this explains why Compound 5 can inhibit the activity of PAD4 (Figure 5).

Since the complex crystal structure of streptonigrin with PAD4 was not available at the time of this work was carried out, we also did molecular docking of streptonigrin and PAD4. Our result shows that streptonigrin has stronger binding to PAD-4 than that of compound 5 with the binding affinity of $-7.2 \mathrm{kcal} / \mathrm{mol}$; however, despite of having more hydrophobicity than compound 5, streptonigrin forms fewer hydrophobic contacts with PAD4. Two PAD4 residues have contacts with streptonigrin, i.e., Trp347 and Val469 and there are two hydrogen bonds between the carboxylic group of streptonigrin and the carboxylic group of Asp305, and oxygen $\mathrm{O}_{8}$ of the carbonyl of streptonigrin with Arg639 (Figure 6). Interestingly, streptonigrin does not form any interaction with Cys645, which raises question whether or not this compounds does interfere the catalytic mechanism and binds covalently to the PAD4 via Cys645 as suggested by Knuckley et al. [9].

To date, only about 40 inhibitors of PAD4 have been reported [32]. Among the reported inhibitors, only 7 compounds are more potent than compound 5 [6-11]. However, compound 5 is the most potent inhibitor discovered by a virtual high throughput screening approach

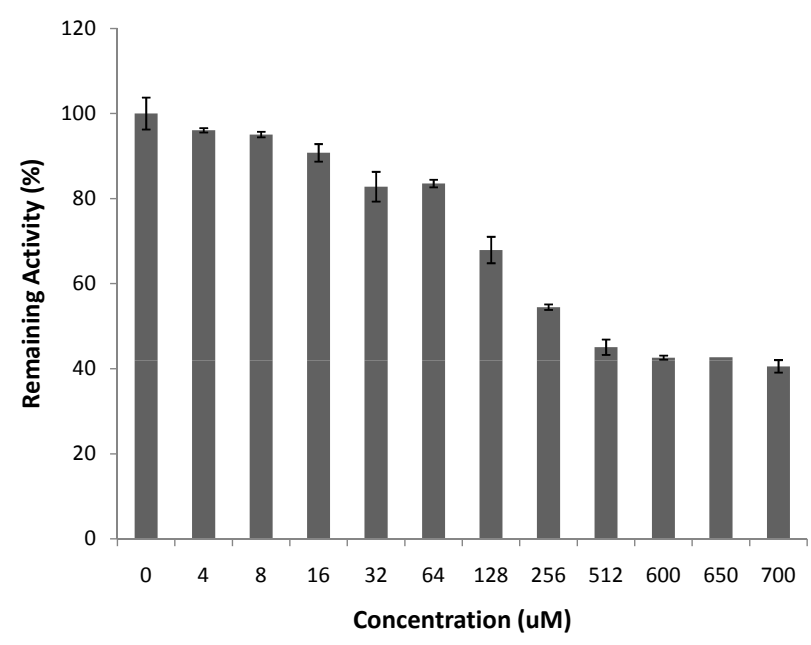

Figure 4: Dose-response curve of compound 5 against PAD4.

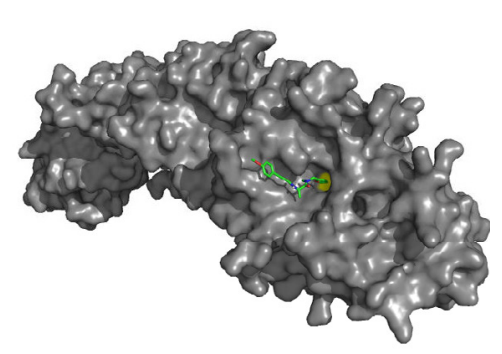

(a)

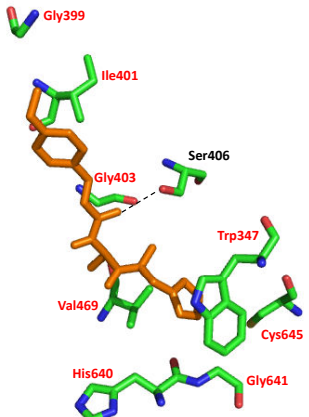

(b)
Figure 5: Binding conformation of compound 5 inside the PAD4 binding pocket. Compound $\mathbf{5}$ is seen occupying the binding pocket with the furan ring is located in proximity with Cys645 (yellow) (a). PAD4 residues with hydrophobic contacts with compound $\mathbf{5}$ (orange, stick structure) are labeled in red, while hydrogen bond is drawn as dashed line (b).

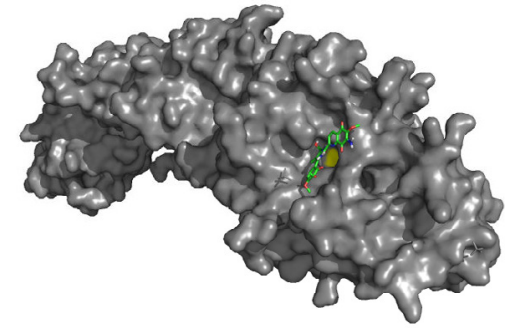

(a)

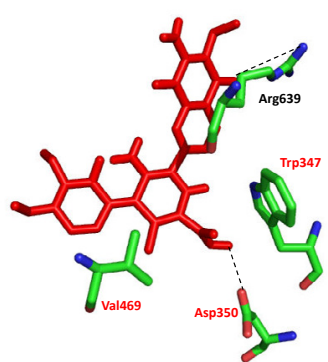

(b)
Figure 6: Binding conformation of streptonigrin inside the PAD4 binding pocket. Streptonigrin is seen occupying the binding pocket with Cys645 in yellow (a). PAD4 residues with hydrophobic contacts with streptonigrin (red, stick structure) are labeled in red, while hydrogen bonds are drawn as dashed line (b).

(vHTS). The discovery of compound $\mathbf{5}$ is more cost-effective, cheap and less time consuming compared to other more potent inhibitors obtained from methods such as high throughput screening (HTS) as vHTS "filtered" millions of compounds and highlighted only the potential compounds. So cost and time were saved for the synthesis and testing of the compounds that could not inhibit PAD4. Common chemotype can be found in compound 5 with inhibitors discovered by structure-based virtual screening in previous study [24]. Compound 5 contains furan ring in the chemical structure which was claimed to be an important feature of the inhibitors reported in previous study [24]. Furan ring is a five-membered aromatic heterocyclic ring that containing in organic compounds widely distributed in nature and often plays important role in various biochemical processes. Its derivatives show biological properties such as antibacterial [33], antispasmodic [34] and anti-inflammatory [35]. Such remarkable properties have made it as an important structural fragment in many pharmaceutical and chemical compounds [36].

\section{Conclusion}

A moderately potent inhibitor for PAD4 has been discovered through ligand-based virtual screening with the application of USR for molecular shape similarity search. Importantly, its core structure is different from that of the parent compound, streptonigrin. Such 
Citation: Teo CY, Abdul Rahman MB, Thean Chor AL, Salleh AB, Ballester PJ, et al. (2013) Ligand-Based Virtual Screening for the Discovery of Inhibitors for Protein Arginine Deiminase Type 4 (PAD4). Metabolomics 3: 118. doi:10.4172/2153-0769.1000118

Page 5 of 5

scaffold hop led to a new class of PAD4 inhibitors. Despite its moderate potency $\left(\mathrm{IC}_{50}\right.$ of $\left.300 \mu \mathrm{M}\right)$, this is a valuable finding in that there are only about 40 known PAD4 inhibitors (none in the nM range) and it is the most potent inhibitor of PAD4 to date found by computational means. Furthermore, this virtual screen was extremely fast and costeffective compared to HTS used for discovery of previously reported PAD4 inhibitors. It is also interesting that the ligand-based protocol used here led to better results than previous structure-based efforts. The compound is commercially available and can now be used as a new starting point for the design of more potent PAD4 inhibitors.

\section{References}

1. Arita K, Hashimoto $H$, Shimizu T, Nakashima K, Yamada M, et al. (2004) Structural basis for $\mathrm{Ca}(2+)$-induced activation of human PAD4. Nat Struct Mol Biol 11: 777-783.

2. Jones JE, Causey CP, Knuckley B, Slack-Noyes JL, Thompson PR (2009) Protein arginine deiminase 4 (PAD4): Current understanding and future therapeutic potential. Curr Opin Drug Discov Devel 12: 616-627.

3. van Boekel MA, Vossenaar ER, van den Hoogen FH, van Venrooij WJ (2002) Autoantibody systems in rheumatoid arthritis: specificity, sensitivity and diagnostic value. Arthritis Res 4: 87-93.

4. Steiner G, Smolen J (2002) Autoantibodies in rheumatoid arthritis and their clinical significance. Arthritis Res 4: S1-S5.

5. Schellekens GA, Visser $\mathrm{H}$, de Jong BA, van den Hoogen FH, Hazes JM, et al (2000) The diagnostic properties of rheumatoid arthritis antibodies recognizing a cyclic citrullinated peptide. Arthritis Rheum 43: 155-163.

6. Luo Y, Arita K, Bhatia M, Knuckley B, Lee YH, et al. (2006) Inhibitors and inactivators of protein arginine deiminase 4: functional and structural characterization. Biochemistry 45: 11727-11736.

7. Luo Y, Knuckley B, Lee YH, Stallcup MR, Thompson PR (2006) A fluoroacetamidine-based inactivator of protein arginine deiminase 4: design, synthesis, and in vitro and in vivo evaluation. J Am Chem Soc 128: 1092-1093.

8. Knuckley B, Luo Y, Thompson PR (2008) Profiling Protein Arginine Deiminase 4 (PAD4): a novel screen to identify PAD4 inhibitors. Bioorg Med Chem 16: 739-745.

9. Knuckley B, Jones JE, Bachovchin DA, Slack J, Causey CP, et al. (2010) A fluopol-ABPP HTS assay to identify PAD inhibitors. Chem Commun (Camb) 46: $7175-7177$

10. Causey CP, Jones JE, Slack JL, Kamei D, Jones LE, et al. (2011) The Development of N-a- (2-Carboxyl)benzoyl-N5-(2-fluoro-1-iminoethyl)-lornithine Amide (o-F-amidine) and N- - -(2-Carboxyl)benzoyl-N5-(2-chloro-1 iminoethyl)-l-ornithine Amide (o-Cl-amidine) As Second Generation Protein Arginine Deiminase (PAD) Inhibitors. J Med Chem 54: 6919-6935.

11. Jones JE, Slack JL, Fang P, Zhang X, Subramanian V, et al. (2012) Synthesis and screening of a haloacetamidine containing library to identify PAD4 selective inhibitors. ACS Chem Biol 7: 160-165.

12. Ballester PJ, Mangold M, Howard NI, Robinson RL, Abell C, et al. (2012) Hierarchical virtual screening for the discovery of new molecular scaffolds in antibacterial hit identification. J R Soc Interface 9: 3196-3207.

13. Mclnnes C (2007) Virtual screening strategies in drug discovery. Curr Opin Chem Biol 11: 494-502.

14. Teo CY, Shave S, Thean Chor A, Salleh A, Abdul Rahman M, et al. (2012) Discovery of a new class of inhibitors for the protein arginine deiminase type 4 (PAD4) by structure- based virtual screening. BMC Bioinformatics 13: S4.

15. Ballester PJ, Finn PW, Richards WG (2009) Ultrafast shape recognition: evaluating a new ligand-based virtual screening technology. J Mol Graph
Model 27: 836-845.

16. Ballester PJ, Richards WG (2007) Ultrafast shape recognition to search compound databases for similar molecular shapes. J Comput Chem 28: 1711 1723

17. Rush TS 3rd, Grant JA, Mosyak L, Nicholls A (2005) A shape-based 3-D scaffold hopping method and its application to a bacterial protein-protein interaction. J Med Chem 48: 1489-1495.

18. Zauhar RJ, Moyna G, Tian L, Li Z, Welsh WJ (2003) Shape signatures: a new approach to computer-aided ligand- and receptor-based drug design. J Med Chem 46: 5674-5690.

19. Schnecke V, Bostrom J (2006) Computational chemistry-driven decision making in lead generation. Drug Discov Today 11: 43-50.

20. Böhm H-J, Flohr A, Stahl M (2004) Scaffold hopping. Drug Discovery Today: Technologies 1: 217-224

21. Issa NT, Kruger J, Byers SW, Dakshanamurthy S (2013) Drug repurposing a reality: from computers to the clinic. Expert Rev Clin Pharmacol 6: 95-97.

22. Ballester PJ, Richards WG (2007) Ultrafast shape recognition for similarity search in molecular databases. Proceedings of the Royal Society A: Mathematical, Physical and Engineering Science 463: 1307-1321.

23. Ballester PJ, Westwood I, Laurieri N, Sim E, Richards WG (2010) Prospective virtual screening with Ultrafast Shape Recognition: the identification of nove inhibitors of arylamine N-acetyltransferases. J R Soc Interface 7: 335-342.

24. Wilson WL, Labra C, Barrist E (1961) Preliminary observations on the use of streptonigrin as an antitumor agent in human beings. Antibiot Chemother 11: 147-150.

25. RJ L (2009) GraFit Version 7I. Erithacus Software Limited.

26. Lenz TL, Hilleman DE (2000) Dofetilide, a new class III antiarrhythmic agent. Pharmacotherapy 20: 776-786.

27. Schneider G, Neidhart W, Giller T, Schmid G (1999) "Scaffold-Hopping" by Topological Pharmacophore Search: A Contribution to Virtual Screening Angew Chem Int Ed Engl 38: 2894-2896.

28. Brown N, Jacoby E (2006) On scaffolds and hopping in medicinal chemistry. Mini Rev Med Chem 6: 1217-1229.

29. Mauser H, Guba W (2008) Recent developments in de novo design and scaffold hopping. Curr Opin Drug Discov Devel 11: 365-374.

30. 30. Sneader W (1996) Drug Prototypes and Their Exploitation: John Wiley \& Sons, USA, 788

31. Lipinski CA, Lombardo F, Dominy BW, Feeney PJ (2001) Experimental and computational approaches to estimate solubility and permeability in drug discovery and development settings. Adv Drug Deliv Rev 46: 3-26.

32. https://www.ebi.ac.uk/chembldb/target/inspect/CHEMBL6111

33. Matsuura H, Saxena G, Farmer SW, Hancock RE, Towers GH (1996) Antibacterial and antifungal polyine compounds from Glehnia littoralis ssp. leiocarpa. Planta Med 62: 256-259.

34. Kupchan SM, Eakin MA, Thomas AM (1971) Tumor inhibitors. 69. Structurecytotoxicity relationships among the sesquiterpene lactones. J Med Chem 14 1147-1152.

35. Meotti FC, Silva DO, Dos Santos AR, Zeni G, Rocha JB, et al. (2003) Thiophenes and furans derivatives: a new class of potential pharmacological agents. Environ Toxicol Pharmacol 15: 37-44.

36. Joule JA, Mills K, Smith GF (1995) Heterocyclic Chemistry: Chapman \& Hall, London, 278.
This article was originally published in a special issue, Drug Repurposing: A New Frontier in Therapeutics handled by Editor(s). Dr. Sivanesan Dakshanamurthy, Georgetown University Medical Center, USA 